\title{
Toward a unified description of hadro- and photoproduction amplitudes
}

\author{
M. W. Paris, R. A. Arndt, R. L. Workman, W. J. Briscoe and I. I. Strakovsky \\ Department of Physics, Data Analysis Center at the Center for Nuclear Studies, The George Washington \\ University, Washington, D.C. 20052, USA
}

\begin{abstract}
The near-term objectives of the research program at the Data Analysis Center are established within the context of the existing partial wave analyses available through the online suite of analysis and database codes accessible through SAID, the Scattering Analysis Interactive Database. This presentation reviews the efforts to determine a model independent method to obtain sets of partial wave amplitudes for strong and electromagnetic reactions, the interpretation of the amplitudes in terms of the excited states of the nucleon, the role of new precision unpolarized and polarized data, and new developments aimed at determining the photoproduction mulitpoles in a unitary, coupled-channel approach. The Chew-Mandelstam technique is discussed and applied to the problem of the $S$-wave pion- and eta-photoproduction amplitudes. The resulting eta production amplitudes exhibit the expected resonant behavior near the eta production threshold. Application of this method to a unified description of the hadro- and photoproduction amplitudes is discussed.
\end{abstract}

Keywords: Formal scattering theory, photoproduction multipoles, Chew-Mandelstam approach, nucleon resonance PACS: $13.60 .-\mathrm{r}, 11.55 . \mathrm{Bq}, 11.80 . \mathrm{Et}, 11.80 . \mathrm{Gw}$

\section{INTRODUCTION}

Non-perturbative features of quantum chromodynamics are crucial in determining the excitation spectrum of the nucleon. The model independent determination of scattering and reaction amplitudes is a necessary component to this end. A range of hadroproduction data, including $\pi N \rightarrow \pi N, \pi N \rightarrow \eta N, \pi N \rightarrow \omega N$, and other inelastic processes used to constrain theoretical models and phenomenological parametrization of the scattering and reaction amplitudes. Currently, however, a renaissance is underway in meson production and resonance physics with reaction data issuing from a number of precision electromagnetic facilities[1, 2, 3, 4, 5, 6, 7]. Phenomenological efforts to analyze these data consistent with some subset of constraints imposed by quantum field theory are under current study. The quality and quantity of data in electromagnetic induced reactions is becoming sufficient to rival and possibly surpass the hadroproduction data. Since the electromagnetic reactions proceed mainly through the hadronic channels, the new data offers the possibility of "back-constraining" the hadronic amplitudes, conventionally determined only in fits to the hadroproduction data.

This presentation describes an exploratory study of the $S$-wave $\pi$ - and $\eta$-photoproduction multipoles in the "ChewMandelstam" approach, related to the $N / D$ representation, to the electromagnetic reaction amplitude. The novel concept, which provokes and permits this exploratory study, is the generalization of the Chew-Mandelstam approach to the electromagnetic sector. We have developed a new form for the amplitude that incorporates multichannel hadronic rescattering effects in a complete manner consistent with unitarity[8]. The near-term objective is to develop a framework in which to analyze the hadro- and electroproduction reactions simultaneously in a global framework.

\section{CHEW-MANDELSTAM PARAMETRIZATION}

Previous work in the determination of the $\eta$ photoproduction amplitudes $[9,10]$ has shown that an approach which includes the coupling of the electromagnetic channel to the $\pi N$ and $\eta N$ channels in the region of energies near the center-of-mass energy, $W=1535 \mathrm{MeV}$ gives a reasonably good description of the data and a plausible form for the amplitudes. However, as our ultimate objective is the simultaneous parametrization of hadro- and photoproduction scattering and reaction observables, we will go beyond the two-channel treatment for this study of the $E_{0+}^{\eta}$ multipole amplitude. 
The form of the Chew-Mandelstam parametrization, which we employ in this study follows as a consequence of the analytic structure imposed by the unitarity[11] of the $S$ matrix in the physical region, $W>m_{i}+m_{t}$, where $W$ is the center-of-mass energy and $m_{i}$ and $m_{t}$ are the masses of the incident and target particles. Confining our attention to two-particle initial and final states, the $S$ matrix is unitary $S^{\dagger} S=S S^{\dagger}=1$. In the partial wave representation with $S=1+2 i \tilde{\rho} T=1+2 i T^{\prime}$ we have

$$
\operatorname{Im} T^{\prime-1}=-\theta\left(W-M_{+}\right),
$$

where $M_{+}$is the diagonal matrix of threshold openings. Since this equation isolates the imaginary part of the inverse- $T$ matrix, we may write

$$
\begin{aligned}
T^{\prime-1} & =\operatorname{Re} T^{\prime-1}+i \operatorname{Im} T^{\prime-1}, \\
& =K^{\prime-1}-i \theta\left(W-M_{+}\right),
\end{aligned}
$$

where we've defined $\operatorname{Re} T^{\prime-1}=K^{\prime-1}$. Multiplying from one side by $T^{\prime}$ and the other by $K^{\prime}$ gives the Heitler integral equation[12]

$$
T^{\prime}=K^{\prime}+K^{\prime} i \theta\left(W-M_{+}\right) T^{\prime} .
$$

This is the starting point for the Chew-Mandelstam parametrization of the reaction amplitude. Equation (1) implies discontinuities in the derivative of the imaginary part at each channel threshold $W=m_{\sigma+}$. The violation of the CauchyRiemann equations at threshold indicates the presence of a branch point.

The partial wave amplitude has the following singularities. There are branch points in the physical region at the channel-opening thresholds as in Eq.(1), branch points in the region $W<0$, and possible poles consistent with causality[13]. An efficient parametrization following Ref.[14], which encodes these singularities, involves the factorization of the partial wave amplitude. This is referred to as the " $N / D$ " approach. We will use the $N / D$ language to clarify the nature of the singularities of the $T$ matrix which are included and those neglected in our Chew-Mandelstam approach.

The preceding discussion of unitarity and the $N / D$ approach provides the context for our present parametrization. The Chew-Mandelstam parametrization developed here is similar to those of Refs.[15]and [16]. We consider Eq.(3) and rewrite it, confining our attention to the $S$ wave multipole as

$$
\begin{aligned}
T^{-1} & =K^{-1}-i \tilde{\rho} \\
& =\left(K^{-1}+\operatorname{Re} C\right)-(\operatorname{Re} C+i \tilde{\rho}) \\
& =\bar{K}^{-1}-C,
\end{aligned}
$$

where $\tilde{\rho}=\rho \theta\left(W-M_{+}\right)$and $\operatorname{Im} C=\tilde{\rho}=\theta\left(W-M_{+}\right) \rho$. The transition matrix is given in terms of the "ChewMandelstam" (CM) $K$ matrix, $\bar{K}$ by

$$
T=\bar{K}+\bar{K} C T
$$

Equation (7) fixes our Chew-Mandelstam parametrization. In the language of the $N / D$ approach, we have neglected the $W<0$ branch points of $N$ and made the approximation $N(W)=\bar{K}(W)$, an entire function. The "ChewMandelstam" function, $C_{\alpha}$ is determined solely by the unitarity constraint, Eq.(1) The Chew-Mandelstam function is given by a Cauchy integral over the discontinuity of $C_{\alpha}$ in the physical region with a single subtraction.

The relationship between the Heitler $K$ matrix and the CM $K$ matrix, $\bar{K}$ is given by

$$
K=\bar{K}+\bar{K}[\operatorname{Re} C] K
$$

This demonstrates a possible advantage of using the CM $K$ matrix. If we consider a polynomial parametrization of a given CM $K$ matrix element then we see, by solving Eq.(8) for $K$

$$
K=\frac{1}{1-\bar{K}[\operatorname{Re} C]} \bar{K}
$$

that poles may appear in the $K$ matrix. Attempts to relate the $K$ matrix poles to resonances have been made[17]. Here, we simply point out that, though $K$ matrix poles are not simply related to $T$ matrix poles[18], Eq.(8) shows that one need not explicitly include pole terms in $\bar{K}$ in order to have poles in $K$. 


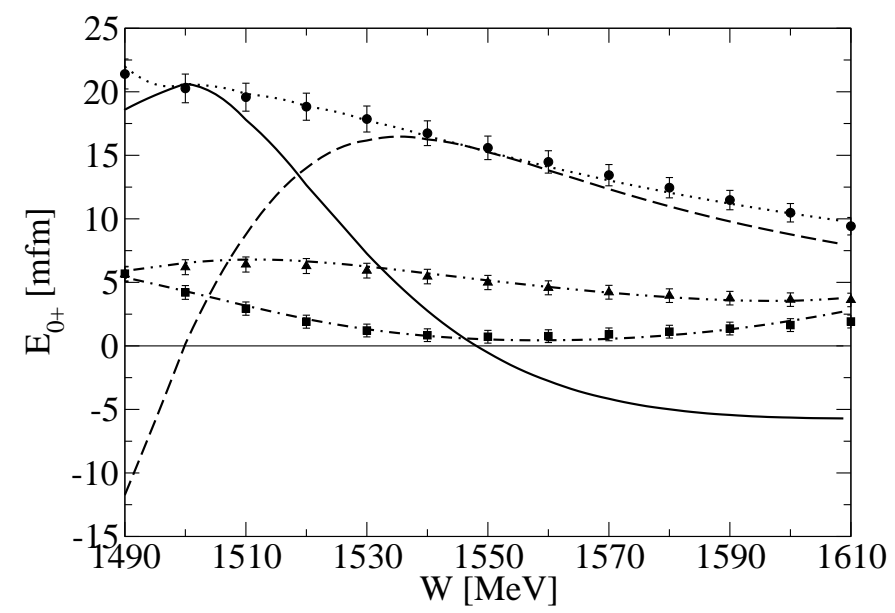

FIGURE 1. The predicted values for the real (solid curve) and imaginary (dashed curve) for $E_{0+}^{\eta}$ versus the energy, $W$. The modulus $\left|E_{0+}^{\eta}\right|$ (dotted curve), the real (dot-dashed curve), and the imaginary (double dot-dashed curve) parts of the $\pi$-photoproduction, $E_{0+}^{\pi}$ were fit to pseudodata generated from the SAID solution[20] with the parametrized form Eq.(11) using 8 parameters.

\section{RESULTS}

The Chew-Mandelstam parametrization for the $T$ matrix, described in the preceding section, has been applied recently[19] to a coupled-channel fit for the $\pi N$ elastic scattering and $\pi N \rightarrow \eta N$ reaction. It gives a realistic description of the data with $\chi^{2}$ per datum better than any other parametrization or model, to our knowledge. The current SAID parametrization used in this fit is given as

$$
T_{\alpha \beta}=\sum_{\sigma}[1-\bar{K} C]_{\alpha \sigma}^{-1} \bar{K}_{\sigma \beta}
$$

where $\alpha, \beta$ and $\sigma$ are channel indices for the considered channels, $\pi N, \pi \Delta, \rho N$ and $\eta N$. Given the success of this approach in the hadronic two-body sector, the application to the study of meson photoproduction is warranted.

The central result of the current exploratory study is to show that this form can be extended to include the electromagnetic channel,

$$
T_{\alpha \gamma}=\sum_{\sigma}[1-\bar{K} C]_{\alpha \sigma}^{-1} \bar{K}_{\sigma \gamma}
$$

where $\gamma$ denotes the electromagnetic channel, $\gamma N$. Note that Eqs.(10) and (11) share the common factor, $[1-\bar{K} C]_{\alpha \sigma}^{-1}$ which encodes, at least qualitatively speaking, the hadronic channel coupling (or rescattering) effects.

The form Eq.(11) for photoproduction should be contrasted with that currently employed in the $\pi$-photoproduction studies of Refs.[20, 10]

$$
T_{\pi \gamma}=A(W)\left(1+i T_{\pi \pi}(W)\right)+B(W) T_{\pi \pi}(W)
$$

where the "structure functions" $A(W)$ and $B(W)$ are parametrized as polynomials in the energy, $W, T_{\pi \gamma}=T_{\pi N, \gamma N}$ and $T_{\pi \pi}=T_{\pi N, \pi N}$, and the factor $A(W)$ contains a contribution from tree-level Born diagrams. This satisfies Watson's theorem[21] (as does Eq.(11)), and is derived via the considerations discussed in Ref.[22]. While resulting in a realistic description of the data and being comparable, at least qualitatively, with other parametrization such as MAID[23] for $\pi$-photoproduction, it does not satisfy the full multichannel unitarity constraint imposed by Eq.(1). This deficiency led us to consider the form in Eq.(11), which manifestly satisfies the multichannel unitarity constraint, Eq.(1).

In the present study we perform a coupled-channel fit of the modulus $\left|E_{0+}^{\eta}(W)\right|$ and (the real and imaginary parts of) the existing SAID and MAID $\pi$-photoproduction amplitudes, $E_{0+}^{\pi}$ in the $S_{11}(1535)$ resonance region. (See Ref.[8] for comparisons to MAID.) The fit was carried out by taking the factor $[1-\bar{K} C]_{\alpha \sigma}^{-1}$ in Eq.(11) as determined in the the hadronic study of Ref.[19] and adjusting the parameters of $\bar{K}_{\sigma \gamma}$ (discussed in detail in the subsections below). The phase of the $E_{0+}^{\eta}$ multipole in this study gives a resonant wave and encourages us to continue with this approach. 
Figure (1) shows the result of fitting the modulus $\left|E_{0+}^{\eta}\right|$ and the real and imaginary parts of the SAID SP09 $E_{0+}^{\pi}$ multipole [6] using an eight-parameter fit. The Chew-Mandelstam $\bar{K}$ matrix was assumed to have four channels, $\pi N, \pi \Delta, \rho N$, and $\eta N$. Eight parameters were varied in the fit to a total of 113 pseudodata points including the modulus $\left|E_{0+}^{\eta}\right|$ over the energy range $1490 \mathrm{MeV} \leq W \leq 1610 \mathrm{MeV}$ and the amplitude $E_{0+}^{\pi}$ over the energy range $1120 \mathrm{MeV}$ $\leq W \leq 1610$. The $\chi^{2}$ per datum over for the fits to the pseudodata, generated with the SAID interactive code facility were less than one in all of the fits made in this work including those in the region $1120 \mathrm{MeV} \leq W \leq 1490 \mathrm{MeV}$ which are not displayed in order to keep the figures manageable and focus attention on the $S_{11}(1535)$ resonance region.

\section{CONCLUSION AND ONGOING WORK}

We reviewed the implication of unitarity on the analytic structure of the single meson production scattering and reaction amplitudes. We related the Chew-Mandelstam $K$-matrix parametrization to the $N / D$ approach, showing that the parametrization of the $\bar{K}$ matrix neglects the effects of the distant left-hand cut. Using the Chew-Mandelstam $K$ matrix $\bar{K}$, we performed a simultaneous coupled-channel fit of the $\eta$-photoproduction $S_{11}$ multipole modulus, $\left|E_{0+}^{\eta}\right|$ and the $\pi$-photoproduction amplitude, $E_{0+}^{\pi}$. The parametrization was restricted only to the CM $K$ matrix elements $\bar{K}_{\sigma \gamma}$ in Eq.(11), while the $[1-\bar{K} C]^{-1}$ factors were taken from the existing SAID fits to the hadronic data. The anticipated resonant structure for the phase of the $E_{0+}^{\eta}$ multipole was demonstrated in fits to SAID amplitudes.

The results of the exploratory study indicate that this is a reasonable approach toward the objective of determining a complete set of scattering and reaction amplitudes for $\pi N \rightarrow \pi N, \pi N \rightarrow \eta N, \gamma N \rightarrow \pi N$, and $\gamma N \rightarrow \eta N$ processes in a multichannel unitary formalism. The ultimate objective of the study will be a simultaneous fit to both the hadronic and electromagnetic scattering and reaction observables and will constitute, at least for two-body unitarity, a global description of the hadro- and photoproduction amplitudes.

\section{ACKNOWLEDGMENTS}

This work is dedicated to the memory of R. Arndt without whom it would not have been possible; it was supported by the US Department of Energy Grant No. DE-FG02-99ER41110.

\section{REFERENCES}

1. J. Ajaka, et al., Phys. Rev. Lett. 81, 1797-1800 (1998).

2. V. Crede, et al., Phys. Rev. Lett. 94, 012004 (2005), hep-ex/ 0311045.

3. T. Nakabayashi, et al., Phys. Rev. C74, 035202 (2006).

4. D. Elsner, et al., Eur. Phys. J. A33, 147-155 (2007), nucl-ex/ 0702032.

5. J. C. McGeorge, et al., Eur. Phys. J. A37, 129-137 (2008), 0711.3443.

6. M. Dugger, et al., Phys. Rev. C79, 065206 (2009), 0903.1110.

7. M. Williams, et al., Phys. Rev. C80, 045213 (2009), 0909.0616.

8. M. W. Paris, and R. L. Workman, Phys. Rev. C82, 035202 (2010), 1004.0455.

9. A. M. Green, and S. Wycech, Phys. Rev. C55, 2167-2170 (1997), nucl-th/ 9703009.

10. R. A. Arndt, A. M. Green, R. L. Workman, and S. Wycech, Phys. Rev. C58, 3636-3640 (1998), nucl-th/ 9807009.

11. W. Zimmerman, Nuovo Cim. 21, 249-273 (1961).

12. W. Heitler, Math. Proc. Camb. Phil. Soc. 37, 291-300 (1941).

13. R. J. Eden, P. V. Landshoff, D. I. Olive, and J. C. Polkinghorne, The Analytic S-matrix, Cambridge University Press, Cambridge, 1966, pp. 231-232.

14. G. F. Chew, and S. Mandelstam, Phys. Rev. 119, 467-477 (1960).

15. J. L. Basdevant, and E. L. Berger, Phys. Rev. D19, 239 (1979).

16. R. A. Arndt, J. M. Ford, and L. D. Roper, Phys. Rev. D32, 1085 (1985).

17. S. Ceci, A. Svarc, B. Zauner, M. Manley, and S. Capstick, Phys. Lett. B659, 228-233 (2008), hep-ph/0611094.

18. R. L. Workman, R. A. Arndt, and M. W. Paris, Phys. Rev. C79, 038201 (2009), 0808.2176.

19. R. A. Arndt, W. J. Briscoe, I. I. Strakovsky, and R. L. Workman, Phys. Rev. C74, 045205 (2006), nucl-th/0605082.

20. R. A. Arndt, R. L. Workman, Z. Li, and L. D. Roper, Phys. Rev. C42, 1853-1863 (1990).

21. K. M. Watson, Phys. Rev. 88, 1163-1171 (1952).

22. R. L. Workman, Phys. Rev. C74, 055207 (2006), nucl-th/ 0510025.

23. W.-T. Chiang, S.-N. Yang, L. Tiator, and D. Drechsel, Nucl. Phys. A700, 429-453 (2002), nucl-th/ 0110034. 